UG-FT-111/99

\title{
Unification through extra dimensions at two loops
}

\author{
M. Masip \\ Dpt. Física Teórica y del Cosmos \\ Universidad de Granada \\ E-18071 Granada, Spain
}

\begin{abstract}
The presence of an extra dimension of size $R \equiv M_{c}^{-1}$ introduces corrections of order $\left(\mu / M_{c}\right) \alpha$ to the gauge and Yukawa couplings and accelerates their running at scales $\mu$ larger than $M_{c}$. This could result in a grand unification scale $M_{X} \approx 20 M_{c}$. We study the corrections at the two-loop level. We find corrections of order $\left(\mu / M_{c}\right) \alpha^{2}$ for the gauge couplings and of order $\left(\mu / M_{c}\right)^{2} \alpha^{2}$ for the Yukawa couplings. Therefore, in the Yukawa sector one and two-loop contributions can be of the same order below $M_{X}$. We show that in the usual scenarios the dominant gauge and Yukawa couplings are decreasing functions of the scale, in such a way that $\left(\mu / M_{c}\right) \alpha$ becomes approximately constant and two-loop contributions introduce just a $30 \%$ correction which does not increase with the scale.
\end{abstract}




\section{Introduction}

String theory, the only known candidate for a fundamental theory including gravity, is formulated in more than three spatial dimensions. Although the extrapolation of the observed physics to higher energies suggests a very small size of the extra dimensions, of $\mathcal{O}\left(M_{\text {Planck }}^{-1}\right)$, it has been recently proposed an alternative framework where some of these dimensions could have a large size [1]. In particular, a compact dimension of inverse radius $R^{-1} \equiv M_{c}=\mathcal{O}(1 \mathrm{TeV})$ where only the gauge bosons and the Higgs fields (but not quarks and leptons) propagate could define a viable scenario within the context of superstring theory [2].

The presence of such a compact dimension at low scales has consequences on the unification of the gauge couplings of the standard model [3]. In the minimal supersymmetric extension of the standard model (MSSM) the observed values are a consequence of the different running from the grand unification scale. Obviously, this picture would be modified by a fifth dimension in the $\mathrm{TeV}$ region. First, we note that 5-dimensional (5D) field theories are not renormalizable and must be taken as effective theories valid only below a more fundamental mass scale, the string (unification) scale $M_{X}$. Second, in order to describe the effective theory we observe that the momentum along a compact dimension is quantized in units of $M_{c}$. This will translate into an infinite tower of Kaluza-Klein (KK) modes associated to any field that penetrates the extra dimension. Below $M_{X}$ it is possible to approximate the theory by a $4 \mathrm{D}$ truncated theory where the high energy modes have been cutoff. In particular, the authors in [3] show that the oneloop corrections to the gauge couplings in the 5D theory almost coincide with the running in the truncated theory. The regularization dependence, which would describe the KK threshold effects, has been discussed in [4].

The KK approach provides a simple framework to understand the running of the couplings. A degree of freedom will be included in the loop only at scales larger than its mass. Since at a given scale $\mu>M_{c}$ the number of KK modes with masses lighter than $\mu$ is $N(\mu)=2 \mu / M_{c}$, the one-loop renormalization group equations (RGEs) become

$$
\frac{\mathrm{d} \alpha_{i}^{-1}}{\mathrm{~d} \ln \mu}=-\frac{1}{2 \pi} b_{i} \rightarrow \frac{\mathrm{d} \alpha_{i}^{-1}}{\mathrm{~d} \ln \mu}=-\frac{1}{2 \pi} b_{i} \frac{2 \mu}{M_{c}},
$$

where $\alpha_{i}=g_{i}^{2} /(4 \pi)$. The first equation gives a linear dependence of $\alpha_{i}^{-1}$ with $\ln \mu$, whereas the second one predicts a much faster exponential (or linear versus $\mu$ ) behaviour.

In addition, the $b_{i}$ coefficients will change as well due to the different field content of a superfield in five dimensions. Actually, the 5D content can be accommodated in complete 4D hypermultiplets of $N=2$ supersymmetry (SUSY) [5]. Each gauge boson will come with its gaugino plus a fermion and a scalar in the adjoint representation of the gauge group, defining a complete vector hypermultiplet (equivalent to a vector plus a chiral supermultiplets). For the matter fields, a chiral hypermultiplet will include a vectorlike pair of chiral superfields. In this way, the two Higgs doublets of the MSSM could in principle be different components of the same 5D field.

Since an extra dimension at a low scale $M_{c}$ would increase exponentially the evolution rate of 
the couplings, gauge unification could occur at much lower energies than in standard scenarios. Unification would not necessarily introduce a hierarchy $M_{X} / M_{E W}=\mathcal{O}\left(10^{14}\right)$ : if $M_{c} \approx M_{E W}$, then $M_{X} / M_{E W}=\mathcal{O}(14)$. The possibility of unification at a scale $M_{X}$ very close to $M_{c}$ has been explored recently by different authors [3, 6]. Their one-loop analysis suggests gauge and also Yukawa coupling unification in several definite models. The behaviour that they obtain above $M_{c}$ is similar to the standard one with the changes $\ln \mu \rightarrow \mu$ and $b_{i} \rightarrow \tilde{b}_{i}$. However, there are important differences in the running when one goes beyond the one-loop level which cannot be accounted with just these substitutions. In particular, whereas the 4D RGEs include at one loop the corrections of type $(\alpha \ln \mu)^{2}$, here the calculation will not include the corrections $(\alpha \mu)^{2}$. Here the parameter in the loop expansion is $\alpha \mu / M_{C}$, not just $\alpha$. Although the fields couple with strength $\alpha$, their number increases linearly with the energy and the analysis remains perturbative only if the product $\alpha \mu / M_{c}$ is small. Then, even if $M_{X} \approx 20 M_{c}$ higher order corrections may be already of order one.

In this paper we analyze the corrections to the one-loop $\beta$-functions of the gauge and the Yukawa couplings in models with an extra dimension'. We show that whereas the gauge sector is protected by the symmetries, in the Yukawa sector the one-loop result is insufficient, since it is modified by higher order corrections proportional to $\left(h_{t}^{2} \mu / M_{c}\right)^{n}$ and $\left(g_{3}^{2} \mu / M_{c}\right)^{n}$. Due to these corrections $h_{t}$ could evolve fast towards large values, which would affect the (two-loop) evolution rate and the perturbative unification of the gauge couplings. In the models analyzed here, however, we observe the opposite behaviour due to the negative sign of the two-loop $\beta$-function, and $h_{t}$ introduces little change in the pattern of gauge unification obtained at one loop.

\section{Two-loop running}

Let us start defining the 5-dim extension of the MSSM. The gauge and Higgs fields will live in the $5 \mathrm{D}$ bulk, whereas the fermions are localized on $4 \mathrm{D}$ boundaries and do not have KK excitations. At $M_{c}$ we find a chiral superfield $\Sigma^{A}$ for each gauge superfield $V^{A}$, whereas in the chiral sector there are pairs $\left(H, H^{\prime}\right)$ of Higgs superfields. Each of these fields has an infinite tower of KK modes of mass $n M_{c}$. The compactification of the fifth dimension must be such that the zero modes define the MSSM, with no massless $\Sigma^{A}$ fields. This usually forces the presence of more than one pair of 5D Higgs fields. To simplify we will consider only one pair of Higgs doublets [3] and comment later on a case with two pairs [8]. We will also assume that (i) the couplings of the 4D chiral fields to the KK excitations coincide with their couplings to the zero modes and (ii) the number of excitations is the usual in a circle. This is not the case for the compactification on the segment $S^{1} / Z_{2}$ [8], with half the resonances in the circle and with the couplings to the heavy modes increased by a factor of $\sqrt{2}$. Our conclusions, however, will be the same in this scenario.

The tower of chiral superfields $\Sigma^{A}$ couples to the chiral Higgses with an effective superpo-

\footnotetext{
1 These corrections to the gauge couplings are in addition to the ones from higher-dimensional operators [7] or massive string modes.
} 
tential of type $W \supset \sqrt{2} g T^{A i}{ }_{j} \Sigma^{A} H_{i} H_{j}^{\prime}$. All the interactions of the quark and lepton fields break the underlying $N=2$ SUSY (their couplings with the heavy Higgs and vector modes do not conserve the momentum along the fifth dimension).

In a general $N=1$ SUSY model with superpotential $W=\frac{1}{6} y_{i j k} \Phi_{i} \Phi_{j} \Phi_{k}$ and gauge symmetry $G$ the $\beta$-functions ( $\beta_{x} \equiv \frac{\mathrm{d} x}{\mathrm{~d} \ln \mu}$, with $x$ a generic gauge or Yukawa coupling) can be deduced from the vector and chiral selfenergies. At one loop they read [9]

$$
\begin{aligned}
\beta_{\tilde{g}}^{(1)} & =\tilde{g}^{3} Q ; \\
\beta_{\tilde{y}_{i j k}}^{(1)} & =\tilde{y}_{i j p} \gamma_{k}^{(1) p}+(k \leftrightarrow i)+(k \leftrightarrow j),
\end{aligned}
$$

where $\tilde{g}=g /(4 \pi), \tilde{y}_{i j k}=y_{i j k} /(4 \pi)$ and

$$
\begin{aligned}
Q & =T(\Phi)-3 C(V) ; \\
\gamma_{j}^{(1) i} & =P_{j}^{i}=\frac{1}{2} \tilde{y}^{i k l} \tilde{y}_{j k l}-2 \tilde{g}^{2} C(\Phi) \delta_{j}^{i} .
\end{aligned}
$$

The group constants are $T(\Phi) \delta_{A B}=\operatorname{Tr}\left(T^{A} T^{B}\right), C(V) \delta_{A B}=f_{A C D} f_{B C D}$ and $C(\Phi) \delta_{j}^{i}=\left(T^{A} T^{A}\right)_{j}{ }_{j}$. In the model under study we have (see Fig. 1) $Q=T(H)+T\left(H^{\prime}\right)+T(\Sigma)-3 C(V)=$ $2 T(H)-2 C(V), P_{H}^{H}=P_{H^{\prime}}^{H^{\prime}}=0$ (due to the cancellation of $V$ and $\Sigma$ contributions), and the MSSM value for the third fermion generation $P_{f}^{f}\left(f=Q, t^{c}, b^{c}, L, \tau^{c}\right)$. Including the sum over all KK modes of mass below $\mu$ we obtain

$$
\begin{aligned}
& \beta_{\tilde{g}_{1}}^{(1)}=\frac{3}{5} \tilde{g}_{1}^{3} \frac{2 \mu}{M_{C}} ; \\
& \beta_{\tilde{g}_{2}}^{(1)}=-3 \tilde{g}_{2}^{3} \frac{2 \mu}{M_{C}} ; \\
& \beta_{\tilde{g}_{3}}^{(1)}=-6 \tilde{g}_{3}^{3} \frac{2 \mu}{M_{C}}
\end{aligned}
$$

and

$$
\begin{aligned}
& \beta_{\tilde{h}_{t}}^{(1)}=\tilde{h}_{t}\left(3 \tilde{h}_{t}^{2}+\tilde{h}_{b}^{2}-\frac{17}{30} \tilde{g}_{1}^{2}-\frac{3}{2} \tilde{g}_{2}^{2}-\frac{16}{3} \tilde{g}_{3}^{2}\right) \frac{2 \mu}{M_{C}} ; \\
& \beta_{\tilde{h}_{b}}^{(1)}=\tilde{h}_{b}\left(3 \tilde{h}_{b}^{2}+\tilde{h}_{t}^{2}-\frac{1}{6} \tilde{g}_{1}^{2}-\frac{3}{2} \tilde{g}_{2}^{2}-\frac{16}{3} \tilde{g}_{3}^{2}\right) \frac{2 \mu}{M_{C}} ; \\
& \beta_{\tilde{h}_{\tau}}^{(1)}=\tilde{h}_{\tau}\left(3 \tilde{h}_{\tau}^{2}-\frac{3}{2} \tilde{g}_{1}^{2}-\frac{3}{2} \tilde{g}_{2}^{2}\right) \frac{2 \mu}{M_{C}},
\end{aligned}
$$

where $\tilde{h}_{t} \equiv \tilde{y}_{Q t^{c} H^{\prime}}, \tilde{h}_{b} \equiv \tilde{y}_{Q b^{c} H}$ and $\tilde{h}_{\tau} \equiv \tilde{y}_{L \tau^{c} H}$.

The generic two-loop $\beta$-functions can be written [9]

$$
\begin{aligned}
\beta_{\tilde{g}}^{(2)} & =2 \tilde{g}^{5} C(V) Q-2 g^{3} r^{-1} C(\Phi) \delta_{j}^{i} P_{i}^{j} ; \\
\gamma_{j}^{(2) i} & =-\left[\tilde{y}^{i m p} \tilde{y}_{j m n}+2 g^{2} C(\Phi) \delta_{j}^{p} \delta_{n}^{i}\right] P_{p}^{n}+2 g^{4} C(\Phi) \delta_{j}^{i} Q,
\end{aligned}
$$

where $r=\delta_{A A}$ is the dimension of the group. 
In our framework the dominant two-loop correction to the gauge couplings would be proportional to $\left(2 \mu / M_{C}\right)^{2}$, coming from two-point diagrams with exchange of heavy KK modes only. However, we have $P_{H}^{H}=P_{H^{\prime}}^{H^{\prime}}=0$ and $P_{\Sigma^{B}}^{\Sigma^{A}}=2 g^{2}[T(H)-C(\Sigma)] \delta_{A B}=g^{2} Q \delta_{A B}$, and the leading contribution to $\beta_{\tilde{g}}^{(2)}$ vanishes [10]. This cancellation reflects the fact that a model with $N=2$ SUSY has to be renormalized only at the one-loop level, i.e., the one-loop counterterms render the theory finite at any order of perturbation [9, 11].

The subleading two-loop correction, proportional to $2 \mu / M_{C}$, comes from diagrams with intermediate chiral fermions or zero modes of gauge or Higgs fields. This correction does not vanish because the underlying $N=2$ SUSY is broken by the interactions of the fields in the loops. It can be written

$$
\begin{aligned}
\beta_{\tilde{g}}^{(2)}= & 2 \tilde{g}^{5} C(V)\left[3\left(T(H)+T\left(H^{\prime}\right)\right)+T(f)+C(\Sigma)-9 C(V)\right] \\
& -2 g^{3} r^{-1} C(H)\left[(4-6) g^{2} C(H) d(H)+\frac{1}{2} y^{H f f^{\prime}} y_{H f f^{\prime}}+\left(H \leftrightarrow H^{\prime}\right)\right] \\
& -2 g^{3} C(\Sigma) 2 g^{2}[2 T(H)-C(\Sigma)] \\
& -2 g^{3} r^{-1} C(f)\left[\frac{1}{2} y^{f k l} y_{f k l}-2 g^{2} C(f) d(f)\right],
\end{aligned}
$$

where $d(\Phi)$ is the dimension of the $\Phi$ representation and $f$ stands for the chiral fermions. The terms in the first line in Eq. (17) come from the diagrams in Fig. 2a [12]. The diagrams with exchange of $(H, V),\left(H^{\prime}, V\right)$ and $V$ fields have a factor of three, since there are three different ways to distribute the momentum along the fifth dimension (i.e., the KK modes) among the fields in the internal loops. For the diagrams with $(f, V)$ and $(\Sigma, V)$ in the loops there is only one possible configuration, corresponding to the exchange of a tower of $V$ and $\Sigma$ fields, respectively. The contributions in the second, third and fourth line in Eq. (7) are due to the diagrams in Figs. 2b,2c,2d, respectively. The diagrams involving $(H, \Sigma)$ have a factor of two and the ones with intermediate $(V, H)$ fields have a factor of 3 . The remaining diagrams have only one possible configuration of KK modes. We obtain

$$
\begin{aligned}
& \beta_{\tilde{g}_{1}}^{(2)}=\tilde{g}_{1}^{3}\left(\frac{199}{25} \tilde{g}_{1}^{2}+\frac{27}{5} \tilde{g}_{2}^{2}+\frac{88}{5} \tilde{g}_{3}^{2}-\frac{26}{5} \tilde{h}_{t}^{2}-\frac{14}{5} \tilde{h}_{b}^{2}-\frac{18}{5} \tilde{h}_{\tau}^{2}\right) \frac{2 \mu}{M_{C}} \\
& \beta_{\tilde{g}_{2}}^{(2)}=\tilde{g}_{2}^{3}\left(\frac{3}{2} \tilde{g}_{1}^{2}+\tilde{g}_{2}^{2}+24 \tilde{g}_{3}^{2}-6 \tilde{h}_{t}^{2}-6 \tilde{h}_{b}^{2}-2 \tilde{h}_{\tau}^{2}\right) \frac{2 \mu}{M_{C}} \\
& \beta_{\tilde{g}_{3}}^{(2)}=\tilde{g}_{3}^{3}\left(\frac{11}{5} \tilde{g}_{1}^{2}+9 \tilde{g}_{2}^{2}-40 \tilde{g}_{3}^{2}-4 \tilde{h}_{t}^{2}-4 \tilde{h}_{b}^{2}\right) \frac{2 \mu}{M_{C}} .
\end{aligned}
$$

In the Yukawa sector the situation is completely different. All the Yukawa interactions break the $N=2$ SUSY structure present in the bulk and allow power-law corrections proportional to $\left(2 \mu / M_{C}\right)^{2}$. These can be especially important if they involve top quark interactions, since $\tilde{h}_{t}^{2}\left(2 \mu / M_{C}\right)$ may be of $\mathcal{O}(1)$ below the unification scale $M_{X}$.

We obtain that the leading corrections cancel in the Higgs selfenergies: $\gamma_{H}^{(2) H}=\gamma_{H^{\prime}}^{(2) H^{\prime}}=$ 0 . For a chiral fermion $f$ the generic expression in (6) becomes

$$
\gamma_{f}^{(2) f}=-\tilde{h}_{f}^{2} P_{f^{c}}^{f^{c}}-2 g^{2} C(f) P_{f}^{f}+2 g^{4} C(f)[2 T(H)+C(\Sigma)-3 C(V)],
$$


where the three contributions have their origin in the diagrams in Figs. 3a,3b,3c, respectively [13]. In particular, for the top quark we find

$$
\begin{aligned}
\gamma_{t}^{(2) t}= & \left(\frac{31}{900} \tilde{g}_{1}^{4}-\frac{9}{4} \tilde{g}_{2}^{4}-\frac{80}{9} \tilde{g}_{3}^{4}+\frac{1}{10} \tilde{g}_{1}^{2} \tilde{g}_{2}^{2}+\frac{8}{45} \tilde{g}_{1}^{2} \tilde{g}_{3}^{2}+8 \tilde{g}_{2}^{2} \tilde{g}_{3}^{2}+\right. \\
& \left.\frac{1}{2} \tilde{g}_{1}^{2} \tilde{h}_{t}^{2}-\frac{3}{2} \tilde{g}_{2}^{2} \tilde{h}_{t}^{2}+\frac{1}{10} \tilde{g}_{1}^{2} \tilde{h}_{b}^{2}-\frac{3}{2} \tilde{g}_{2}^{2} \tilde{h}_{b}^{2}-2 \tilde{h}_{t}^{4}-2 \tilde{h}_{b}^{4}\right)\left(\frac{2 \mu}{M_{C}}\right)^{2} ; \\
\gamma^{(2) t^{c}}= & \left(\frac{184}{225} \tilde{g}_{1}^{4}-\frac{80}{9} \tilde{g}_{3}^{4}+\frac{128}{45} \tilde{g}_{1}^{2} \tilde{g}_{3}^{2}-\tilde{g}_{1}^{2} \tilde{h}_{t}^{2}+3 \tilde{g}_{2}^{2} \tilde{h}_{t}^{2}-2 \tilde{h}_{t}^{4}-2 \tilde{h}_{t}^{2} \tilde{h}_{b}^{2}\right)\left(\frac{2 \mu}{M_{C}}\right)^{2} .
\end{aligned}
$$

Analogous expressions can be found for the bottom quark and the tau lepton. The dominant terms in the two-loop Yukawa $\beta$-functions are then

$$
\begin{aligned}
\beta_{\tilde{h}_{t}}^{(2)=} & \tilde{h}_{t}\left(\frac{767}{900} \tilde{g}_{1}^{4}-\frac{9}{4} \tilde{g}_{2}^{4}-\frac{160}{9} \tilde{g}_{3}^{4}+\frac{1}{10} \tilde{g}_{1}^{2} \tilde{g}_{2}^{2}+\frac{136}{45} \tilde{g}_{1}^{2} \tilde{g}_{3}^{2}+8 \tilde{g}_{2}^{2} \tilde{g}_{3}^{2}-\right. \\
& \left.\frac{1}{2} \tilde{g}_{1}^{2} \tilde{h}_{t}^{2}+\frac{3}{2} \tilde{g}_{2}^{2} \tilde{h}_{t}^{2}+\frac{1}{10} \tilde{g}_{1}^{2} \tilde{h}_{b}^{2}-\frac{3}{2} \tilde{g}_{2}^{2} \tilde{h}_{b}^{2}-4 \tilde{h}_{t}^{4}-2 \tilde{h}_{b}^{4}-2 \tilde{h}_{t}^{2} \tilde{h}_{b}^{2}\right)\left(\frac{2 \mu}{M_{C}}\right)^{2} ; \\
\beta_{\tilde{h}_{b}}^{(2)}= & \tilde{h}_{b}\left(\frac{167}{900} \tilde{g}_{1}^{4}-\frac{9}{4} \tilde{g}_{2}^{4}-\frac{160}{9} \tilde{g}_{3}^{4}+\frac{1}{10} \tilde{g}_{1}^{2} \tilde{g}_{2}^{2}+\frac{8}{9} \tilde{g}_{1}^{2} \tilde{g}_{3}^{2}+8 \tilde{g}_{2}^{2} \tilde{g}_{3}^{2}+\right. \\
& \left.\frac{1}{2} \tilde{g}_{1}^{2} \tilde{h}_{t}^{2}-\frac{3}{2} \tilde{g}_{2}^{2} \tilde{h}_{t}^{2}-\frac{1}{10} \tilde{g}_{1}^{2} \tilde{h}_{b}^{2}+\frac{3}{2} \tilde{g}_{2}^{2} \tilde{h}_{b}^{2}-2 \tilde{h}_{t}^{4}-4 \tilde{h}_{b}^{4}-2 \tilde{h}_{t}^{2} \tilde{h}_{b}^{2}\right)\left(\frac{2 \mu}{M_{C}}\right)^{2} ; \\
\beta_{\tilde{h}_{\tau}}^{(2)=} & \tilde{h}_{\tau}\left(\frac{303}{100} \tilde{g}_{1}^{4}-\frac{9}{4} \tilde{g}_{2}^{4}+\frac{9}{10} \tilde{g}_{1}^{2} \tilde{g}_{2}^{2}+\frac{9}{10} \tilde{g}_{1}^{2} \tilde{h}_{\tau}^{2}+\frac{3}{2} \tilde{g}_{2}^{2} \tilde{h}_{\tau}^{2}-4 \tilde{h}_{\tau}^{4}\right)\left(\frac{2 \mu}{M_{C}}\right)^{2} .
\end{aligned}
$$

\section{Numerical results}

For the gauge couplings, the two loops introduce corrections $\mathcal{O}\left(\tilde{g}_{i}^{2}+\tilde{h}_{i}^{2}\right)$ to the one-loop result (the tilde stands for the coupling over $4 \pi$ ). For example, keeping only the top and the color couplings we have $\beta_{\tilde{g}_{3}}^{(2)} / \beta_{\tilde{g}_{3}}^{(1)} \approx 6.6 \tilde{g}_{3}^{2}+0.6 \tilde{h}_{t}^{2}$. In the Yukawa sector the two-loop corrections are $\mathcal{O}\left[\left(\tilde{g}_{i}^{2}+\tilde{h}_{i}^{2}\right)\left(2 \mu / M_{c}\right)\right]$. In particular, for $h_{t}$ we have $\beta_{\tilde{h}_{t}}^{(2)} / \beta_{\tilde{h}_{t}}^{(1)} \approx\left(2 \mu / M_{c}\right)\left(-17.7 \tilde{g}_{3}^{4}-4 \tilde{h}_{t}^{4}\right) /\left(-5.3 \tilde{g}_{3}^{2}+\right.$ $\left.3 \tilde{h}_{t}^{2}\right)$. For an initial value $\tilde{h}_{t}=\tilde{g}_{3}=1 /(4 \pi), \beta_{\tilde{h}_{t}}^{(2)} / \beta_{\tilde{h}_{t}}^{(1)}=1$ for $\mu \approx 9 M_{c}$.

This estimate shows that the unification of the gauge and Yukawa couplings at $\approx 20 M_{c}$ cannot be stablished from just a one-loop calculation, it requires the analysis of higher order corrections. It could happen, for example, that the two-loop corrections increase the value of $h_{t}$ with the scale and make it very large at energies just above $9 M_{c}$. This would also affect the evolution rate of the gauge couplings and could spoil de gauge unification obtained at one loop.

In the particular scenario under study, however, we obtain the opposite effect. At $M_{c}$ the one-loop $\beta$-functions for $\tilde{g}_{3}$ and $\tilde{h}_{t}$ are both negative, which retards the scale where the one and two-loop $\beta$-functions become of the same order. In addition, the two-loop contribution to $\beta_{\tilde{h}_{t}}$ is also negative, further decreasing $\tilde{h}_{t}$ and pushing up the range of energies where $\beta_{\tilde{h}_{t}}^{(2)} / \beta_{\tilde{h}_{t}}^{(1)}$ is small. We plot in Fig. 4 the numerical running (including all the couplings) for $\tan \beta=2$ and $M_{c}=1 \mathrm{TeV}$. Dashes correspond to the one-loop running, so one can see graphically the 
effect of second order corrections. At $\mu \approx 10 \mathrm{TeV}$ two-loop effects account for a $25 \%$ of the $\beta$-functions (see Fig. 5). This relative value of $\beta_{\tilde{h}_{2}}^{(2)}$, however, does not increase since the linear

behaviour of $\beta_{\tilde{h}_{2}}^{(2)}$ with $\mu$ is dumped by the running of $\tilde{h}_{t}$ to smaller values dictated by the sign of its $\beta$-function.

We have also deduced the RGE in the model compactified in the orbifold $S^{1} / Z_{2}$. This model includes just one mode at each KK level, two pairs of Higgs $\left(H, H^{\prime}\right) \mathrm{KK}$ towers, and a factor of $\sqrt{2}$ in the couplings of the chiral fermions to the massive KK modes. Gauge unification is lost but the behaviour of two-loop corrections is completely analogous. We obtain (see Fig. 6) also negative values for $\beta_{\tilde{h}_{t}}^{(1)}$ and $\beta_{\tilde{h}_{t}}^{(2)}$, which again avoids large couplings at scales just above $\mu \approx 10 M_{c}$.

\section{Conclusions}

We have studied whether in models with a compact dimension the low-energy value of the gauge and Yukawa couplings can be related perturbatively with the value at a scale $\mu>M_{c}$. In particular, we have calculated the two-loop corrections in a model with gauge unification at $M_{X} \approx 20 M_{c}$ and also in the model compatified on $S^{1} / Z_{2}$.

For the gauge couplings we obtain that the underlying $N=2$ SUSY in the KK sector cancels the leading contributions in the two-loop $\beta$-functions. In consequence, $\beta_{g_{i}}^{(2)} / \beta_{g_{i}}^{(1)}=\mathcal{O}(\alpha / 4 \pi)$, where $\alpha$ refers to a generic $g_{i}^{2} /(4 \pi)$ or $h_{f}^{2} /(4 \pi)$.

In the Yukawa sector there is no symmetry reason for cancellations and $\beta_{h_{f}}^{(2)} / \beta_{h_{f}}^{(1)}=\mathcal{O}[(\alpha / 4 \pi)$ $\left.\left(2 \mu / M_{c}\right)\right]$. An estimate suggests that the one and the two-loop contributions to $\beta_{h_{t}}$ have the same size for $\mu \approx 10 M_{c}$. In this particular model, however, the sign of $\beta_{h_{t}}$ is always negative, what makes $h_{t}$ a decreasing function of $\mu$ and widens the range of energies with perturbative values of $h_{t}^{2}\left(\mu / M_{c}\right)$. Actually, at two-loops all the gauge and the Yukawa couplings except for $\alpha_{1}$ decrease with the scale in such a way that the product $\alpha \mu$ is approximately constant at $\mu \gg M_{c}$. We obtain that at $\mu=10 M_{c} \beta_{h_{t}}^{(2)} / \beta_{h_{t}}^{(1)} \approx 0.3$. Whether or not higher order corrections could change substantially the two-loop result is then unclear: a factor of 10 from the diagramatics at the next order could make $\beta_{h_{t}}^{(3)} \approx \beta_{h_{t}}^{(1)}$.

In summary, our analysis shows that in models with an extra dimension the one-loop running of Yukawa couplings is clearly insufficient, since higher order corrections can be just as important at scales few times above $M_{c}$. Although this type of large corrections vanish in the running of the gauge couplings, to claim unification one needs to make sure that $h_{t}$ stays perturbative up to the unification scale. In the particular models studied here the negative sign of the one and two-loop $\beta_{h_{t}}$ suggests that this is the case. But, since the expansion parameter is $\mathcal{O}(0.3)$ much below $M_{X}$, gauge unification could still be spoiled by higher order corrections. 


\section{Acknowledgements}

The author thanks J. Prades for discussions. This work has was supported by CICYT under contract AEN96-1672 and by the Junta de Andalucía under contract FQM-101. 


\section{References}

[1] N. Arkani-Hamed, S. Dimopoulos and G. Dvali, Phys. Lett. B429 (1998) 263; I. Antoniadis, N. Arkani-Hamed, S. Dimopoulos and G. Dvali, Phys. Lett. B436 (1998) 257.

[2] I. Antoniadis, Phys. Lett. B246 (1990) 377; I. Antoniadis and K. Benakli, Phys. Lett. B326 (1994) 69; Z. Kakushadze and S.-H.H. Tye, Nucl. Phys. B548 (1999) 180.

[3] K.R. Dienes, E. Dudas, T. Gherghetta, Phys. Lett. B436 (1998) 55; Nucl. Phys. B537 (1999) 47.

[4] J. Kubo, H. Terao, G. Zoupanos, hep-ph/9910277.

[5] M. Sohnius, Phys. Rep. 128 (1985) 39.

[6] D. Ghilencea and G.G. Ross, Phys. Lett. B442 (1998) 165; Z. Kakushadze, Nucl. Phys. B548 (1999) 205; S.A. Abel and S.F. King, Phys. Rev. D59 (1999) 095010; T. Kobayashi, J. Kubo, M. Mondragón and G. Zopaunos, Nucl. Phys. B550 (1999) 99; C.D. Carone, Phys. Lett. B454 (1999) 70; P.H. Frampton and A. Rašin, Phys. Lett. B460 (1999) 313; A. Delgado and M. Quirós, Nucl. Phys. B559 (1999) 235; D. Dumitru and S. Nandi, hep-ph/9906514.

[7] H-C. Cheng, B.A. Dobrescu and C.T. Hill, hep-ph/9906327.

[8] A. Pomarol and M. Quirós, Phys. Lett. B438 (1998) 255; A. Delgado, A. Pomarol and M. Quirós, hep-ph/9812489.

[9] D.R.T. Jones and L. Mezincescu, Phys. Lett. B136 (1984) 242; I. Jack and D.R.T. Jones, Phys. Lett. B333 (1994) 372.

[10] Z. Kakushadze and T.R. Taylor, hep-th/9905137.

[11] P.S. Howe, K.S. Stelle and P.C. West, Phys. Lett. B124 (1983) 55.

[12] A. Parkes and P. West, Phys. Lett. B138 (1984) 99.

[13] P. West, Phys. Lett. B137 (1984) 371. 

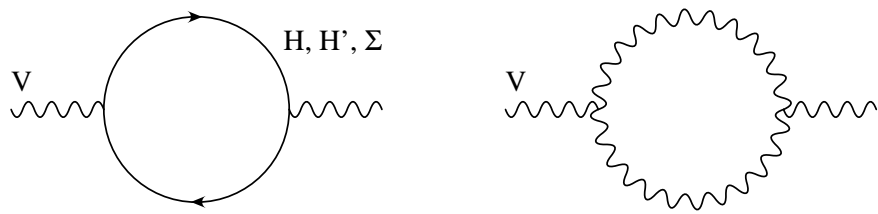

(a)
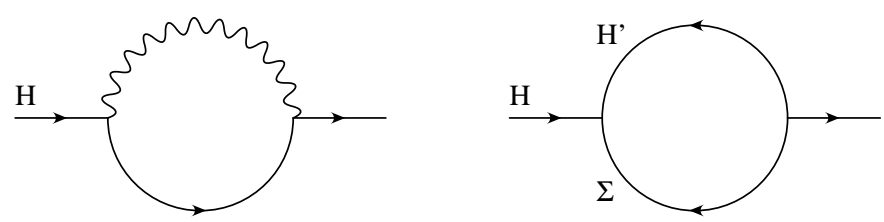

(b)
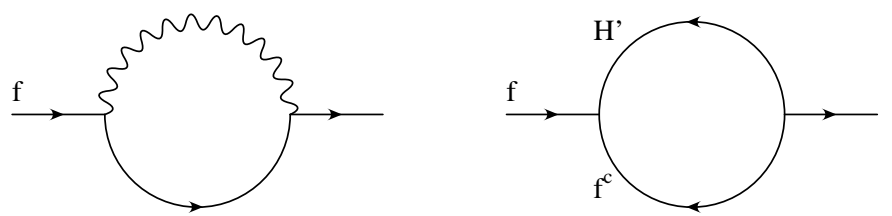

(c)

Figure 1: One-loop contributions to the vector, Higgs and fermion selfenergies. Solid (wiggle) lines indicate chiral (vector) supermultiplets. Ghost contributions are not included. The two diagrams in Fig. 1(b) cancel. 

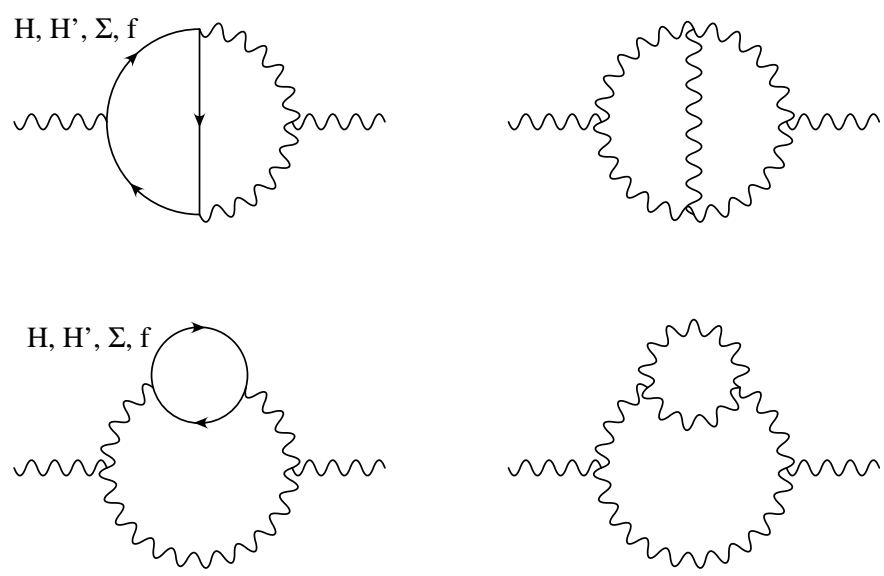

(a)
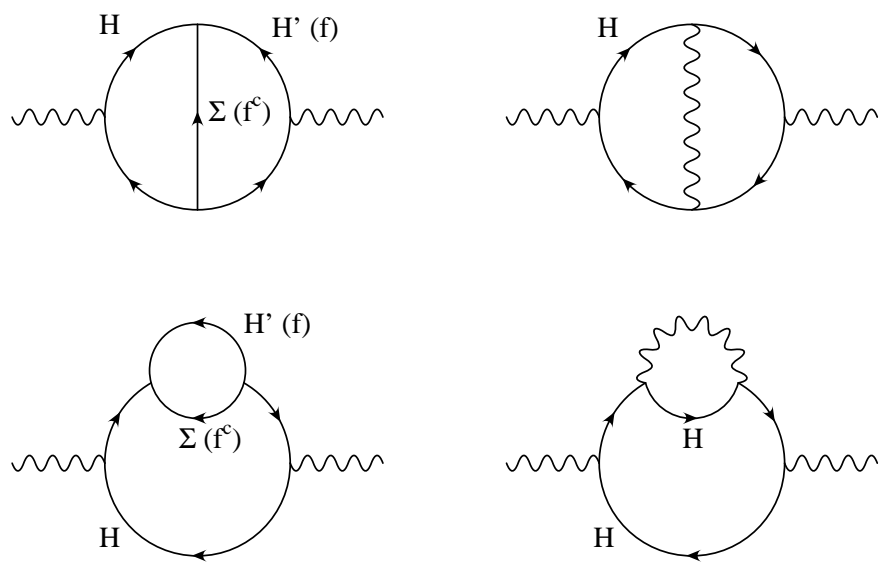

(b)
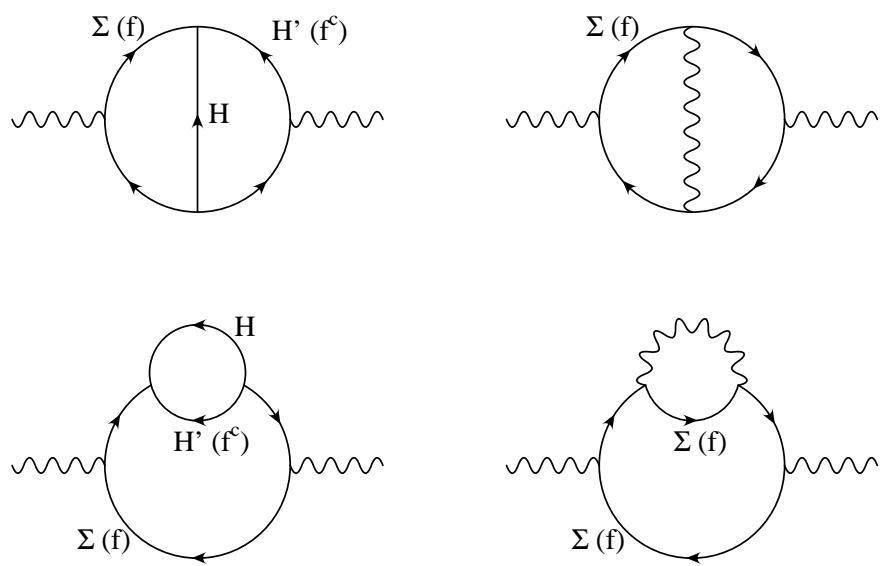

(c)

Figure 2: Two-loop contributions $\left(\propto \mu / M_{c}\right)$ to the vector selfenergy. Counterterm and ghost contributions are not included. 

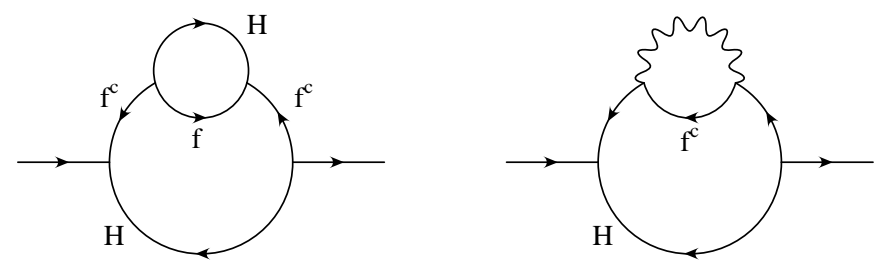

(a)
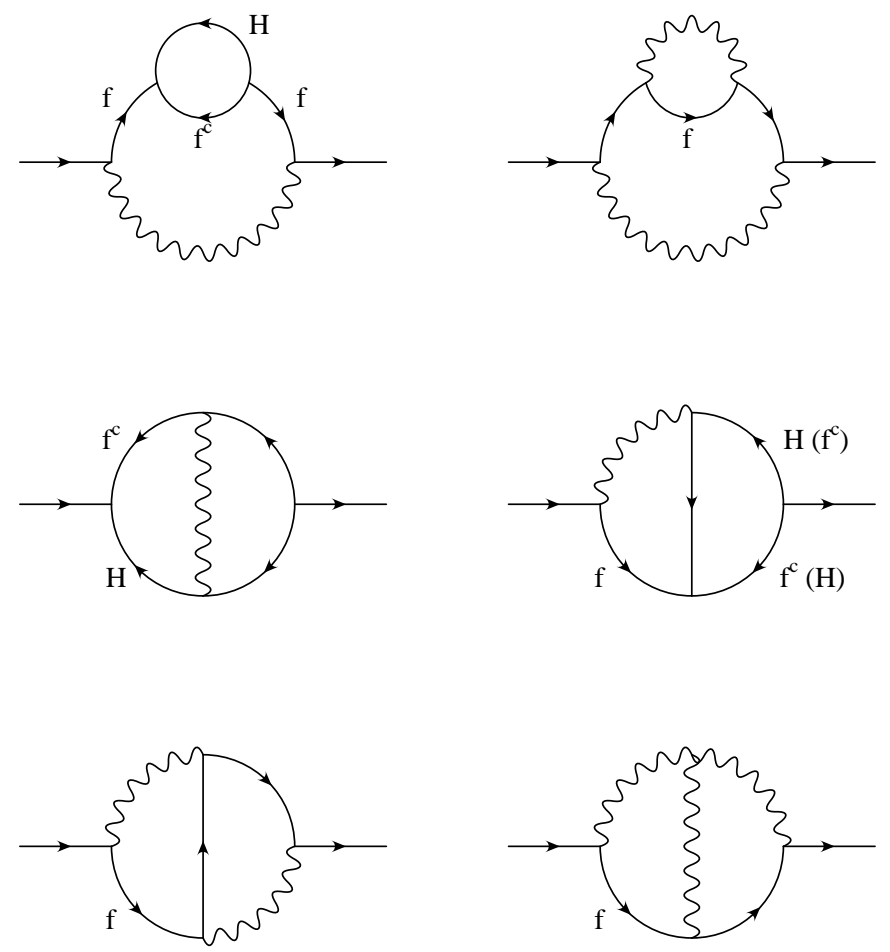

(b)
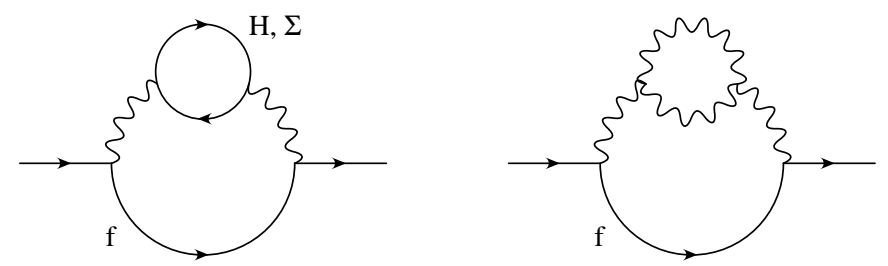

(c)

Figure 3: Two-loop contributions $\left(\propto \mu^{2} / M_{c}^{2}\right)$ to the fermion selfenergies. 


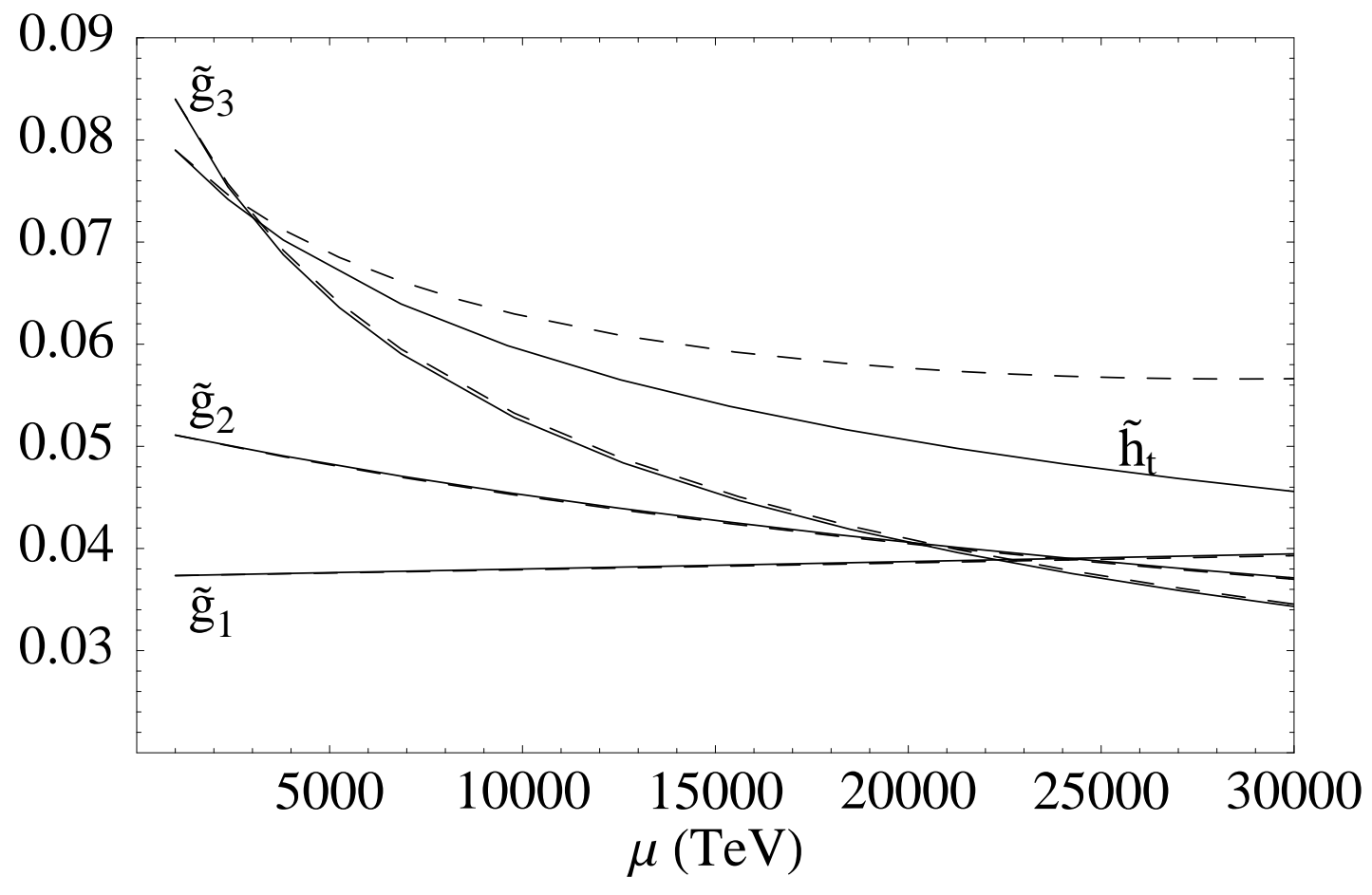

Figure 4: One-loop (dashed) and two-loop (solid) running of gauge and Yukawa couplings for the minimal model with $\tan \beta=2$ and $M_{c}=1 \mathrm{TeV}$. 


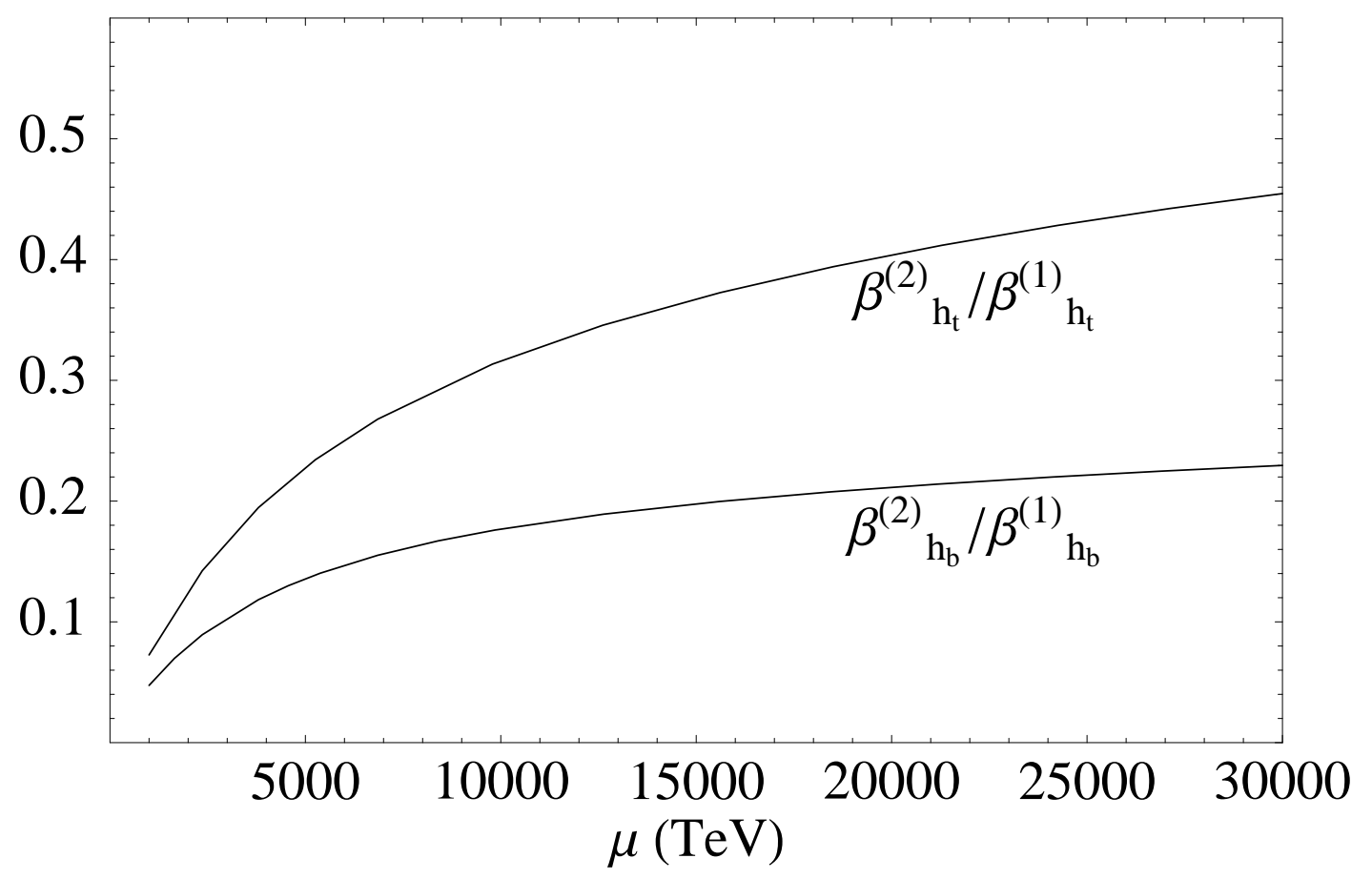

Figure 5: Absolut value of $\beta_{\tilde{h}_{t}}^{(2)} / \beta_{\tilde{h}_{t}}^{(1)}$ and $\beta_{\tilde{h}_{b}}^{(2)} / \beta_{\tilde{h}_{b}}^{(1)}$ for the model in Fig. 4. 


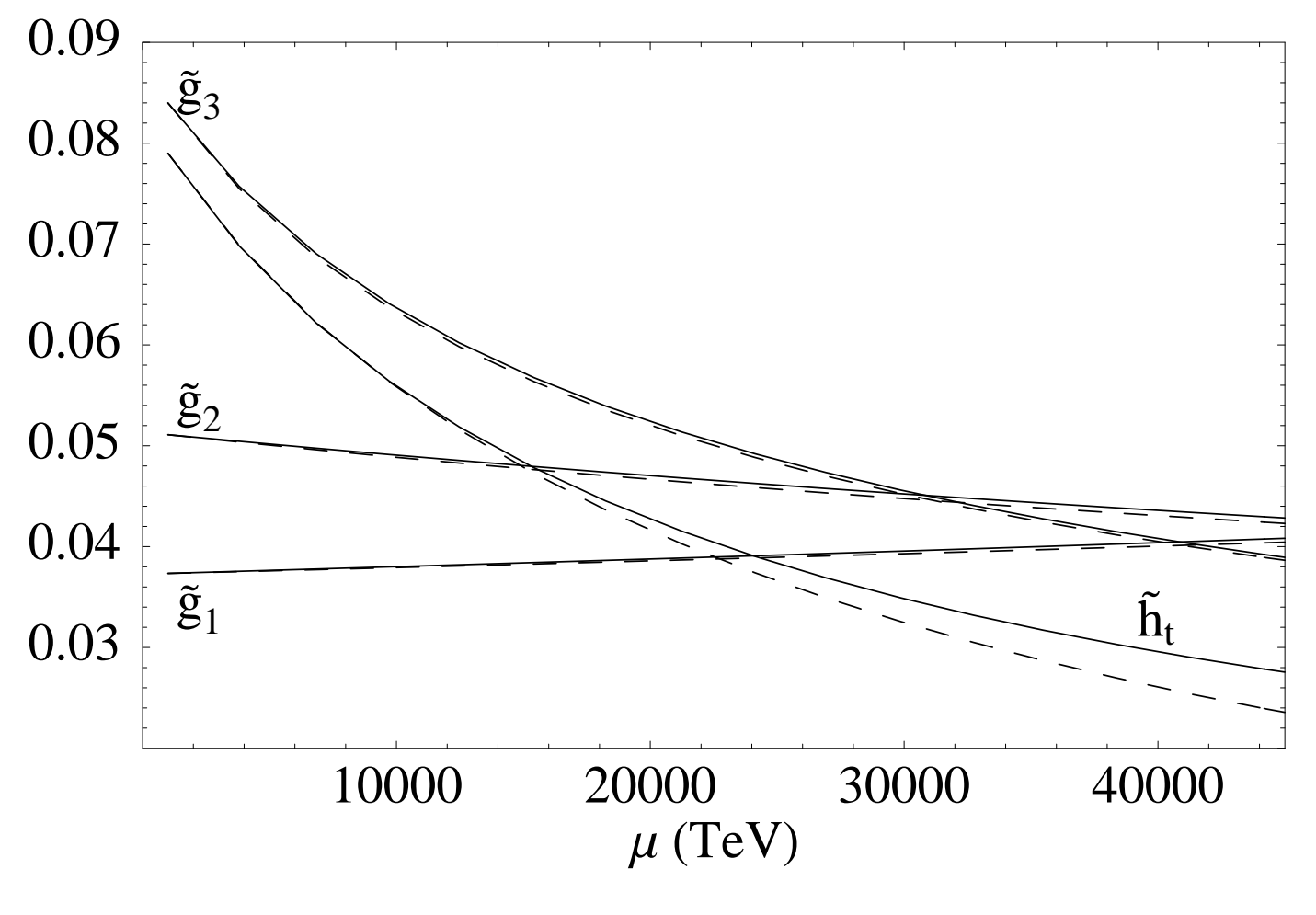

Figure 6: One-loop (dashed) and two-loop (solid) running of gauge and Yukawa couplings for the model compactified on $S^{1} / Z_{2}$ with $\tan \beta=2$ and $M_{c}=1 \mathrm{TeV}$. 